\title{
Conventional molecular dynamics and metadynamics simulation studies of the binding and unbinding mechanism of TTR stabilizers AG10 and tafamidis
}

\author{
Shuangyan Zhou ${ }^{1}$, Siyu Ge1, Wenying Zhang ${ }^{1}$, Qiyuan Zhang ${ }^{1}$, Shuai \\ Yuan $^{1 *}$, Glenn V. Lo ${ }^{2}$, Yusheng Dou ${ }^{2}$
}

1 Chongqing Key Laboratory on Big Data for Bio Intelligence, Chongqing university of posts and telecommunications, Chongqing 400065, China

2 Department of Chemistry and Physical Sciences, Nicholls State University, P.O.

$$
\text { Box 2022, Thibodaux, LA 70310, USA }
$$

*Corresponding author

Email address: yuanshuai@,cqupt.edu.cn 

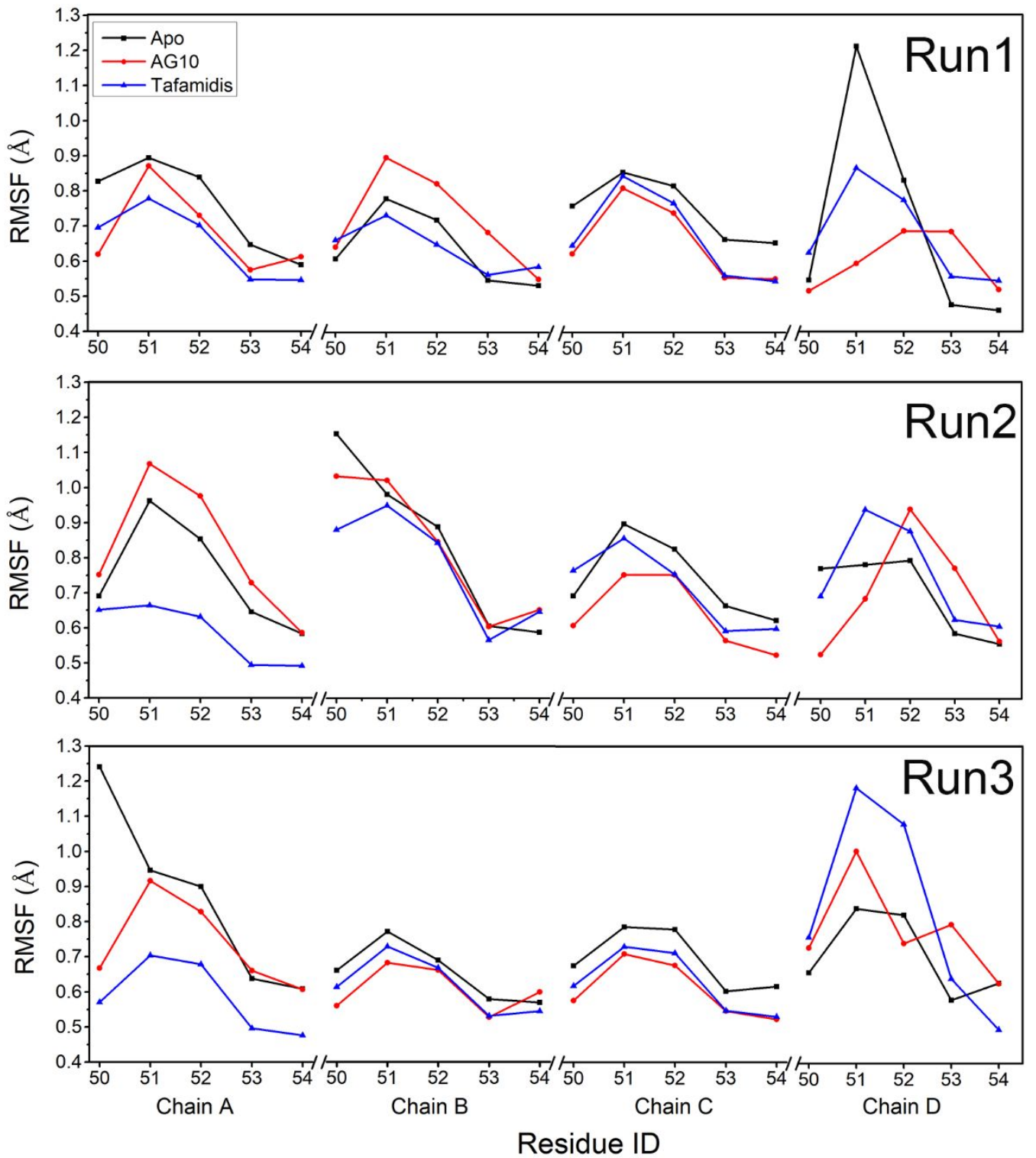

Figure S1. The calculated root-mean-squre fluctuations (RMSFs) of C $\alpha$ atom around the CD loop of each chain from residue 50 to 54 . Three parallel runs of each system were calculated, marked as Run1, Run2 and Run3, respectively. 
(a)
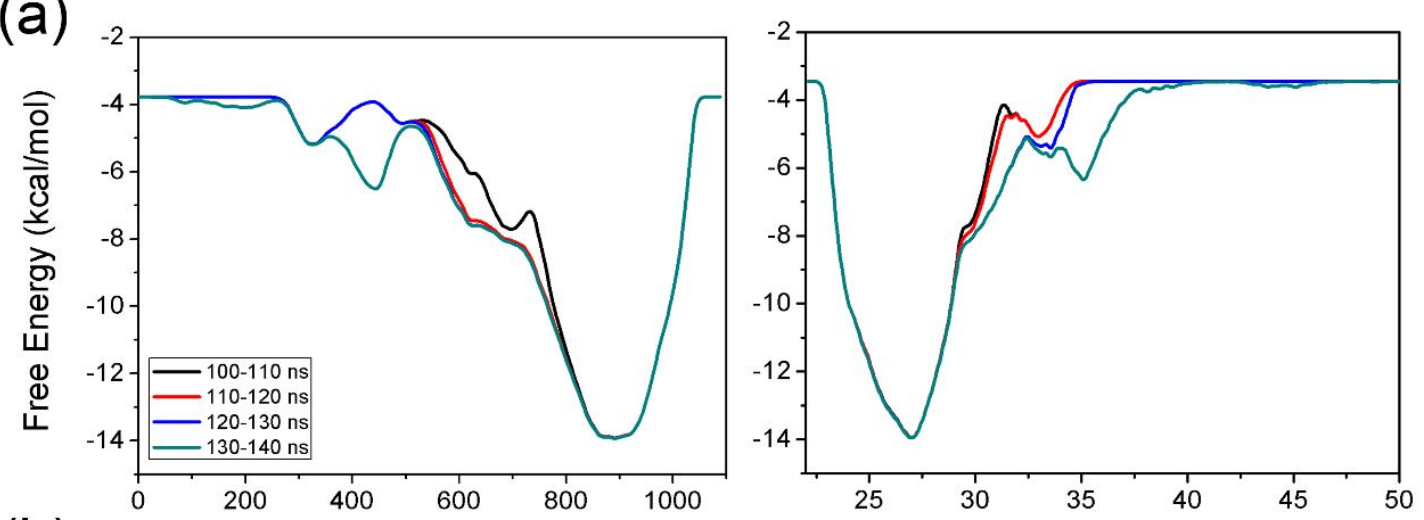

(b)

Coordination Number
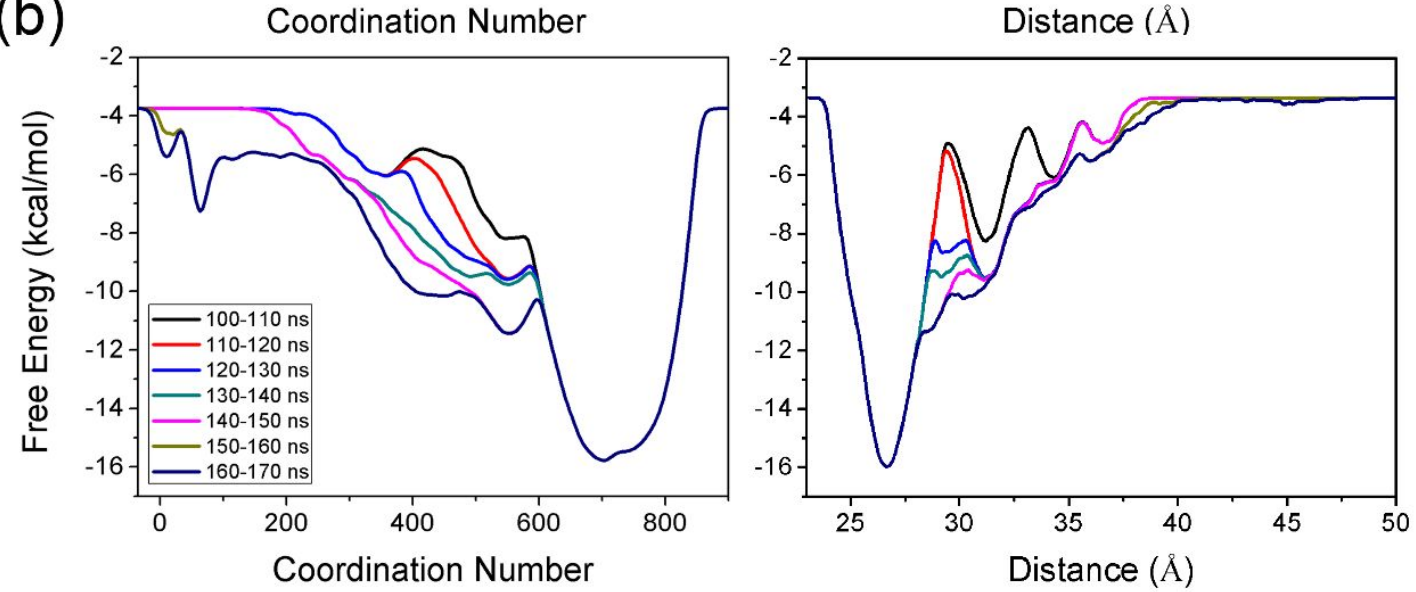

Figure S2. Convergence examination of metadynamics simulation for (a) AG10

dissociation and (b) tafamidis dissociation. 

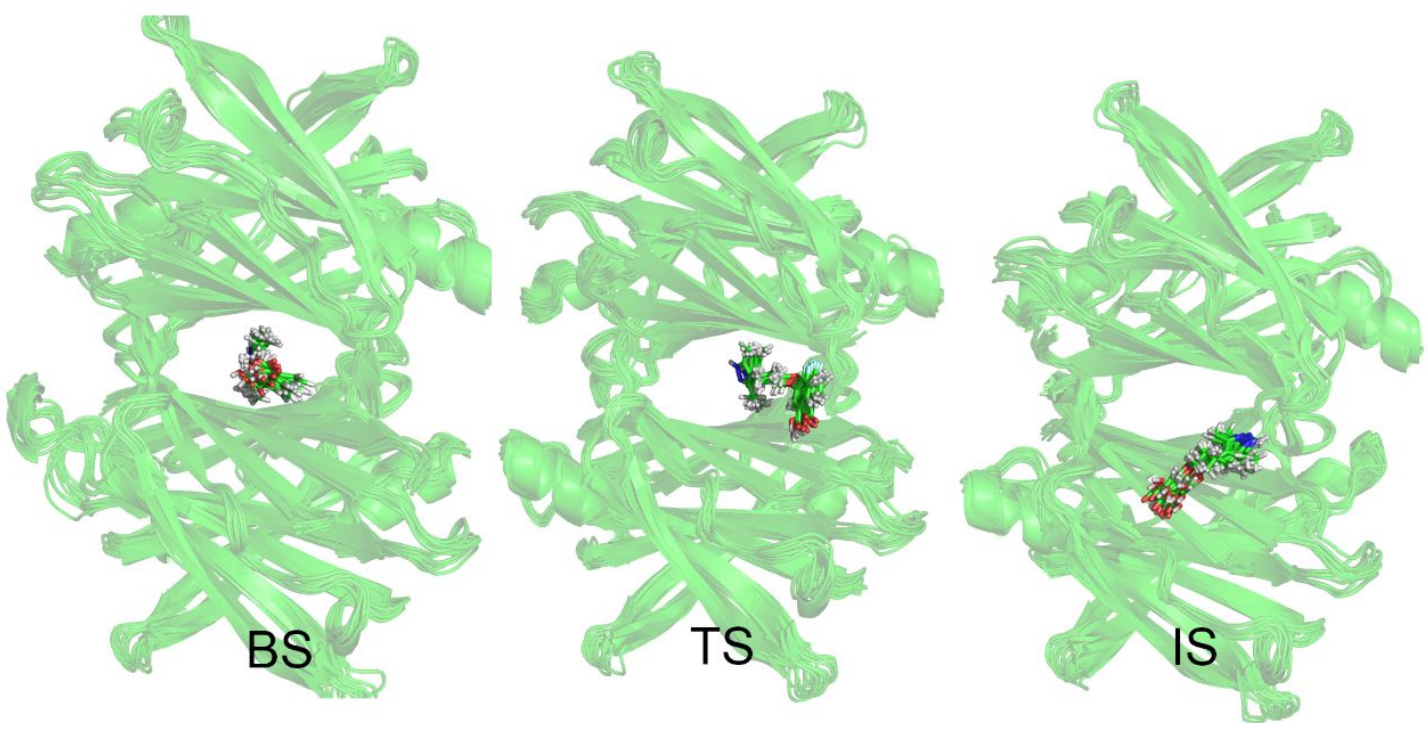

Figure S3. The superposed representative structures of each state during the unbindg process of AG10 


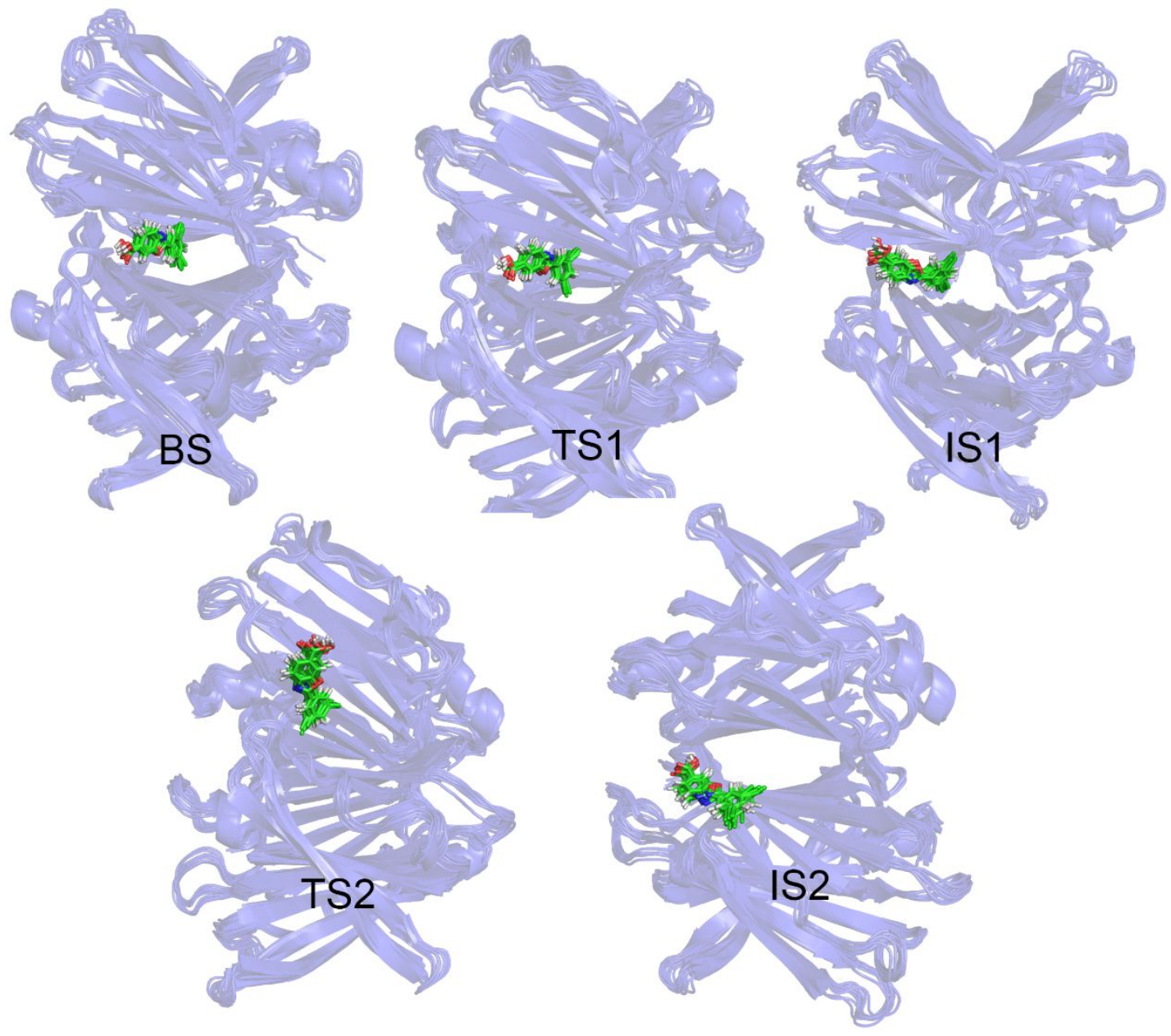

Figure S4. The superposed representative structures of each state during the unbindg process of tafamidis. 


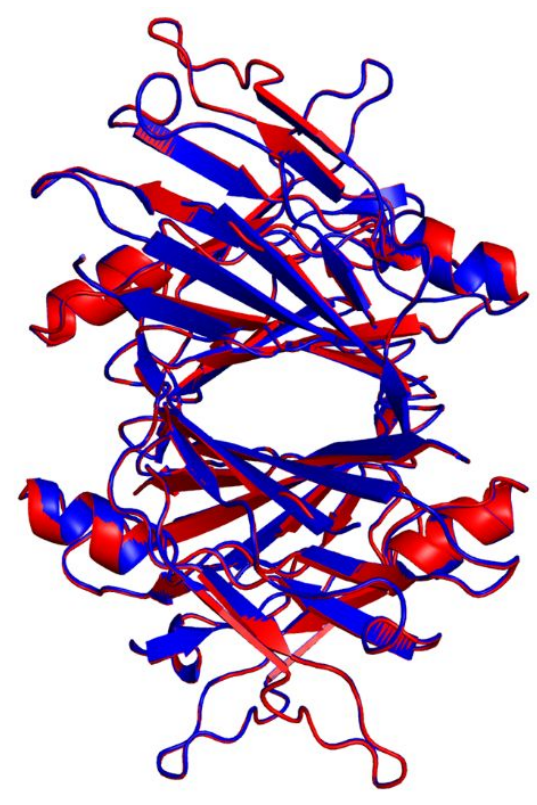

Figure S5. The superposed TTR tetramer structures of 4HIS (blue) and 3TCT (red).

The RMSD for 3TCT reference to 4HIS is about $0.127 \AA$. 

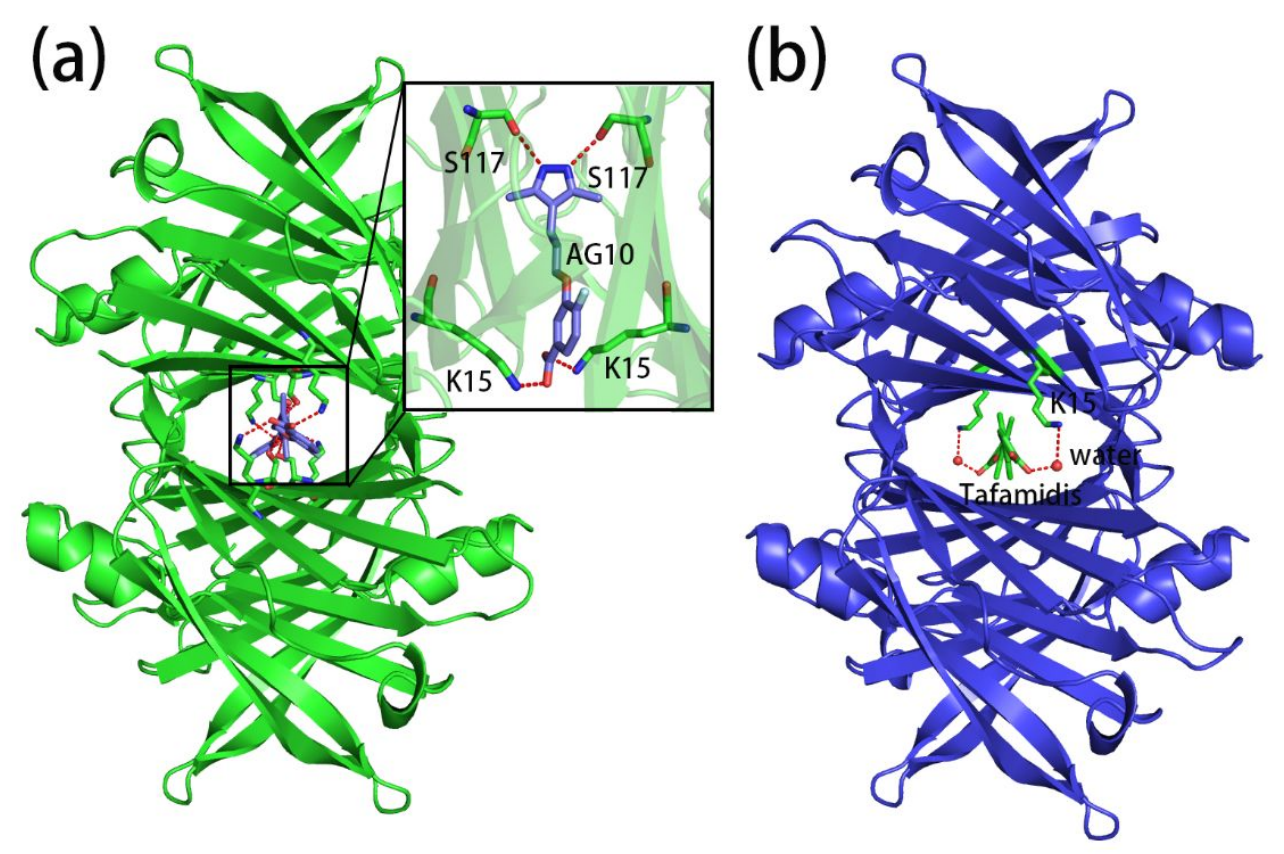

Figure S6. The initial binding models of (a) AG10 and (b) tafamidis. 


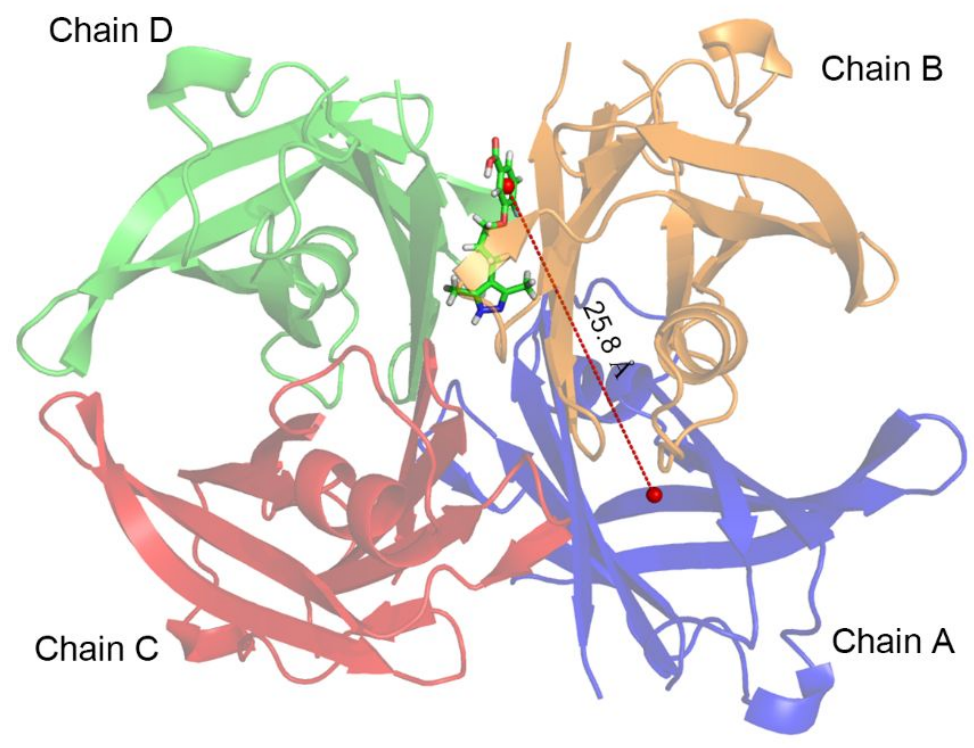

Figure S7. The selected coordinate of the distance between the center mass of aromatic ring of AG10 and center mass of chain A of TTR tetramer. 